\title{
Bootstrapping QCD Using Pion Scattering Amplitudes
}

\author{
Andrea L. Guerrieri \\ Instituto de Física Teórica, UNESP, ICTP South American Institute for Fundamental Research, \\ Rua Dr Bento Teobaldo Ferraz 271, 01140-070 São Paulo, Brazil \\ João Penedones \\ Institute of Physics, École Polytechnique Fédérale de Lausanne (EPFL), Rte de la Sorge, \\ BSP 728, CH-1015 Lausanne, Switzerland \\ Pedro Vieira \\ Instituto de Física Teórica, UNESP, ICTP South American Institute for Fundamental Research, \\ Rua Dr Bento Teobaldo Ferraz 271, 01140-070, São Paulo, Brazil and Perimeter Institute for Theoretical Physics, \\ 31 Caroline St $N$ Waterloo, Ontario N2L 2Y5, Canada
}

(Received 29 November 2018; published 19 June 2019)

\begin{abstract}
We consider the $S$-matrix bootstrap of four-dimensional scattering amplitudes with $O(3)$ symmetry and no bound states. We explore the allowed space of scattering lengths which parametrize the interaction strength at threshold of the various scattering channels. Next we consider an application of this formalism to pion physics. A signature of pions is that they are derivatively coupled leading to (chiral) zeros in their scattering amplitudes. In this work we explore the multidimensional space of chiral zeros positions, scattering length values, and resonance mass values. Interestingly, we encounter lakes, peninsulas, and kinks depending on which sections of this intricate multidimensional space we consider. We discuss the remarkable location where QCD seems to lie in these plots, based on various experimental and theoretical expectations.
\end{abstract}

DOI: 10.1103/PhysRevLett.122.241604

Introduction and setup.-Pions are approximate Goldstone bosons for spontaneous chiral symmetry breaking in QCD. In this Letter we study the following question: Do pion scattering amplitudes take a special place in the space of consistent $S$ matrices?

We assume isospin symmetry, which is a very good approximation of the physical world. To wit we consider pions as particles of mass $m_{\pi}=1$ in the vector representation of $O(3)$ so that their 2 to 2 scattering amplitude reads

$$
\begin{aligned}
\mathcal{T}_{a b}^{c d}= & A(s \mid t, u) \delta_{a b} \delta^{c d}+A(t \mid s, u) \delta_{a}^{c} \delta_{b}^{d} \\
& +A(u \mid s, t) \delta_{a}^{d} \delta_{b}^{c},
\end{aligned}
$$

where $s, t, u=4-s-t$ are the usual Mandelstam invariants. Crossing symmetry is simply $A(s \mid t, u)=A(s \mid u, t)$. The partial wave expansion is given by

Published by the American Physical Society under the terms of the Creative Commons Attribution 4.0 International license. Further distribution of this work must maintain attribution to the author(s) and the published article's title, journal citation, and DOI. Funded by SCOAP ${ }^{3}$.

$$
\begin{aligned}
\mathcal{T}= & (3 A(s \mid t, u)+A(t \mid s, u)+A(u \mid s, t)) \mathbb{P}_{0} \\
& +(A(t \mid s, u)-A(u \mid s, t)) \mathbb{P}_{1}+(A(t \mid s, u)+A(u \mid s, t)) \mathbb{P}_{2} \\
= & \frac{16 \pi i \sqrt{s}}{\sqrt{s-4}} \sum_{I=0,1,2} \mathbb{P}_{I} \sum_{\ell}(2 \ell+1)\left(1-S_{\ell}^{(I)}(s)\right) P_{\ell}\left(\frac{u-t}{u+t}\right),
\end{aligned}
$$

where $P_{\ell}$ are Legendre polynomials and $\mathbb{P}_{I}$ are the $(s$-channel) projectors onto the three possible isospin channels. $(I=0$ for singlet, $I=1$ for vector, and $I=2$ for symmetric traceless tensor). Because of the absence of bound states, we consider the following analytic and crossing symmetric ansatz [1]:

$A(s \mid t, u)=\sum_{n \leq m}^{\infty} a_{n m}\left(\rho_{t}^{n} \rho_{u}^{m}+\rho_{t}^{m} \rho_{u}^{n}\right)+\sum_{n, m}^{\infty} b_{n m}\left(\rho_{t}^{n}+\rho_{u}^{n}\right) \rho_{s}^{m}$,

where $\rho_{z} \equiv([\sqrt{8 / 3}-\sqrt{4-z}] /[\sqrt{8 / 3}+\sqrt{4-z}])$ is a conformal mapping of the $z$ complex plane minus the cut $z>4$ to the $\rho$ unit disk [2]. In the partial wave decomposition, unitarity reads simply $\left|S_{\ell}^{(I)}(s)\right| \leq 1$ for $s>4$. To extract $S_{\ell}^{(I)}(s)$ we project the amplitude as usual, by multiplying by the appropriate Legendre polynomial and integrating over the scattering angle. This is a 
linear operation so that all the $S_{\ell}^{(I)}(s)$ can be explicitly written as linear combinations of the constants $a_{n m}$ and $b_{n m}$.

For numerical explorations we replace $\infty$ in (2) by some large number $N_{\max }$. Then we can simply explore the space of possible $S$ matrices by extremizing various physical observables subject to the unitarity constraints while making sure the values converge as we increase the cutoff $N_{\max }$ as well as other numerical cutoffs such as the number of grid points where we check unitarity and how many spins we impose it on; see [1] for details [5].

So far this is totally general and valid for any unitary relativistic quantum theory with $O(3)$ vector particles. To zoom in on pions we need further input. A lot is known about pions as nicely reviewed in [6-10].

First of all we have experimental data. There are clear resonances in the $\operatorname{spin} \ell=1$ and $\operatorname{spin} \ell=2$ corresponding to the so-called $\rho$ and $f_{2}(1270)$ particles and a broader resonance for $\operatorname{spin} \ell=0$ corresponding to the $\sigma$ particle. A sharp characterization of these resonances is as zeros of the corresponding partial waves. For example, the $\rho$ particle resonance will have a complex mass $m_{\rho}$ such that [11]

$$
S_{1}^{(1)}\left(m_{\rho}^{2}\right)=0 .
$$

Let us emphasize that a free theory has $S_{\ell}^{(I)}=1$ so a resonance is quite a strong effect, very far from a free theory. For pions, we do have two very important points located at subthreshold values of $s$ where the weak coupling conditions (often referred to as Adler's zeros [13])

$$
S_{0}^{(0)}\left(s_{0}\right)=1 \quad \text { and } \quad S_{0}^{(2)}\left(s_{2}\right)=1
$$

hold. The same weak coupling conditions can be imposed as zeros in the corresponding partial wave amplitudes as it follows from their definition $S_{\ell}^{(I)}=1+2 i \sqrt{1-4 / s} \mathcal{T}_{\ell}^{(I)}$. Indeed, leading order chiral perturbation theory [14] predicts

$$
\mathcal{T}_{0}^{(0)}=\frac{2 s-1}{32 \pi f_{\pi}^{2}}, \quad \mathcal{T}_{0}^{(2)}=\frac{2-s}{16 \pi f_{\pi}^{2}}, \quad \mathcal{T}_{1}^{(1)}=\frac{s-4}{96 \pi f_{\pi}^{2}},
$$

where $f_{\pi}$ is the pion decay constant. From this we read off the tree-level predictions $s_{0}=1 / 2$ and $s_{2}=2$.

Finally, at low energy we have the expansion of the partial wave amplitudes close to threshold

$$
\operatorname{Re}\left[\mathcal{T}_{\ell}^{(I)}\right]=k^{2 \ell}\left[a_{\ell}^{(I)}+b_{\ell}^{(I)} k^{2}+\mathcal{O}\left(k^{4}\right)\right]
$$

where $k=\sqrt{s / 4-1}$ is the center-of-mass momentum, $a_{\ell}^{(I)}$ are the scattering lengths, and $b_{\ell}^{(I)}$ are the effective ranges. In Table I we summarize their experimental values.

Numerical explorations.-We first ask what is the allowed region in the three-dimensional space of scattering lengths $\left\{a_{0}^{(0)}, a_{1}^{(1)}, a_{0}^{(2)}\right\}$. It turns out they are all bounded
TABLE I. Experimental determination of scattering lengths and Roy equations results for effective ranges of $\pi-\pi$ scattering up to spin one $[10,15,16]$.

\begin{tabular}{lll}
\hline \hline I & \multicolumn{1}{c}{$\mathcal{O}\left(k^{0}\right)$} & \multicolumn{1}{c}{$\mathcal{O}\left(k^{2}\right)$} \\
\hline 0 & $a_{0}^{(0)}=0.2196 \pm 0.0034$ & $b_{0}^{(0)}=0.276 \pm 0.006$ \\
2 & $a_{0}^{(2)}=-0.0444 \pm 0.0012$ & $b_{0}^{(2)}=-0.0803 \pm 0.0012$ \\
1 & & $a_{1}^{(1)}=0.038 \pm 0.002$ \\
\hline \hline
\end{tabular}

from below [17]. [18] The boundary of the allowed region is shown in Fig. 1. It has a smooth tip, a point of highest curvature; we do not know if it corresponds to any physical theory. The black dot represents the QCD experimental values from Table I. We see that it is well inside the allowed region. Indeed, we can study the various phase shifts of the extremal solutions along the boundary and realize these do not resemble QCD. This is not surprising because we are not imposing anything yet about the chiral symmetry breaking physics which pions describe.

The lake.-To do so, we would like to impose the existence of the two chiral zeros described in (4). However, these zeros appear in the unphysical region $s<4$ and their position cannot be measured experimentally. Our next investigation aims at getting some hints about their position.

We start by fixing one chiral zero $s_{0}$ in the singlet channel amplitude $\mathcal{T}_{0}^{(0)}(s)$ and the vector $\rho$ resonance with mass parameters $m_{\rho} \simeq(5.5+0.5 i)$. [23] Then we maximize and minimize the symmetric channel amplitude $\mathcal{T}_{0}^{(2)}(s)$ for $0<s<4$ obtaining the blue solid curves in Fig. 2(a) (here represented as $s_{0}=1 / 2$ ). We learn that there

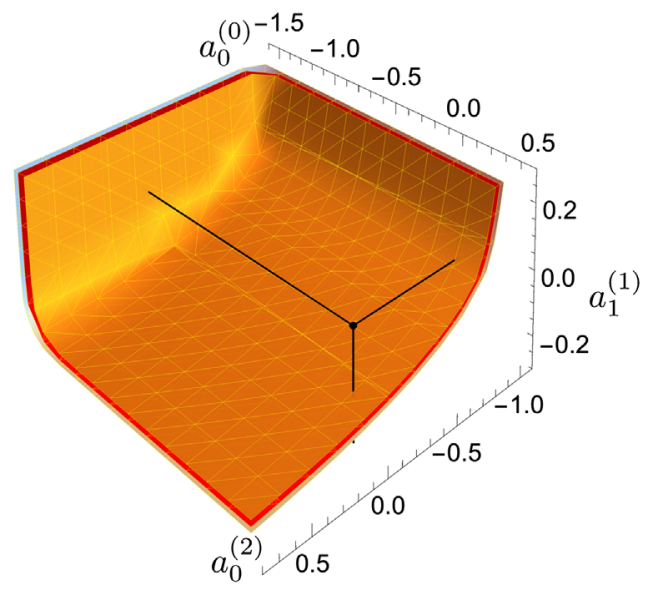

FIG. 1. Exploration of the minimum values scattering lengths can take. The three surfaces here correspond to $N_{\max }=12,14,16$ (orange, red, light blue). The fact they are almost indistinguishable is the sign of their very good convergence. The QCD values (Table I) are represented by a dot here (the errors are smaller than the size of the dot) and are of course well within the allowed region where scattering lengths live. 


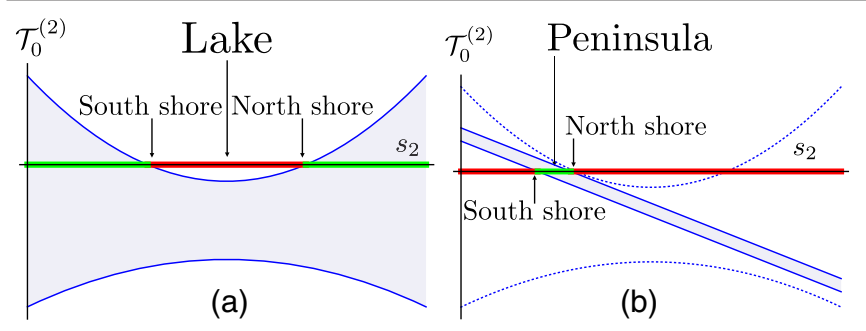

FIG. 2. Schematic picture of the lake (a) and of the peninsula determination (b). (a) Solid blue lines enclose the allowed region for the amplitude $\mathcal{T}_{0}^{(2)}$ when we impose the chiral zero condition at $s_{0}=1 / 2$ and the $\rho$ resonance. The same region is shown in (b) by dashed blue lines. Solid blue lines in (b) embrace the allowed region when the three scattering lengths are set to the experimental values within errors. In both panels we denote in red the region where we cannot fix $s_{2}$ and in green where we can.

are regions where we cannot impose a chiral zero $s_{2}$ since the maximum and minimum are both negative there (red segment). Therefore, we can simultaneously impose the weak coupling conditions $\mathcal{T}_{0}^{(2)}\left(s_{2}\right)=0$ and $\mathcal{T}_{0}^{(0)}\left(s_{0}\right)=0$ only when the upper boundary is positive (green segment). Repeating this game for various $s_{0}$ allows us to exclude a full region in the $\left(s_{0}, s_{2}\right)$ plane. This region, depicted in Fig. 3, is what we dub the pion Lake.

Notice that, while the amplitude maximizing $\mathcal{T}_{0}^{(2)}(s)$ is unique, there can be many amplitudes having the same zero $s_{2}$. On the other hand, the intersection of the upper boundary of the allowed region with the $s_{2}$ axis selects a unique amplitude since a zero there coincides with the maximum possible value at that point. This uniqueness of the boundary theories is the same sort of uniqueness found and thoroughly explored in the conformal bootstrap [24].

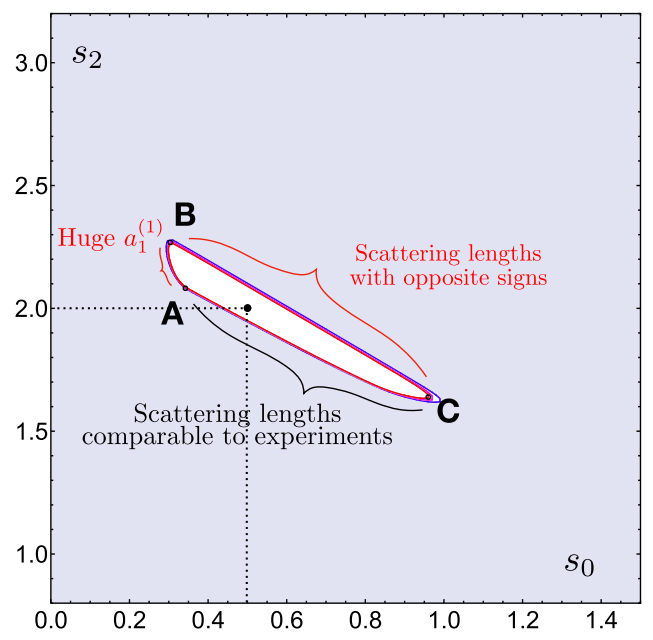

FIG. 3. Pion lake: the white region is the exclusion area in the plane $\left(s_{0}, s_{2}\right)$ when we fix the $\rho$ resonance. The black point corresponds to tree-level chiral perturbation theory (5) which is now excluded. We show the shape of the lake for three different $N_{\max }=12,13,14$ (blue to red): convergence is guaranteed by the three curves almost overlapping.
Remarkably, the prediction $\left(s_{0}, s_{2}\right)=(1 / 2,2)$ of leading order chiral perturbation theory (5) is clearly excluded. What the lake is telling us is that we need to be considerably far from that weak coupling point to be able to include such a strong coupling phenomena as a resonance. Therefore, we can think that the boundary of the lake corresponds to theories that are as free as possible given that they contain the $\rho$ resonance [25].

If we were to take the complex $\rho$ mass to be larger and larger (i.e., further away in the Mandelstam plane) the lake would become thinner and thinner eventually becoming a line segment passing through the chiral perturbation theory point and very well fitted by our numerics to $s_{2}-2+4 / 5\left(s_{0}-1 / 2\right)=0$. Curiously this line of zeros would correspond to a tree-level amplitude $A(s \mid t, u) \propto$ $s-m_{\pi}^{2} \alpha ; \alpha=1$ yields (5). It would be interesting to further explore this line segment analytically; it should be related to an interesting line of perturbative field theories.

Nicely, along the south shore, the sign of the scattering lengths match the experimental one being also close in magnitude to them (see the left inset in Fig. 4).

The peninsula.-Motivated by these explorations, we consider a third exploratory game where we now impose the $\rho$ resonance condition plus the three scattering length inequalities $\left|a_{0}^{(0)}-0.2196\right|<0.034$ etc. given in Table I. These upper and lower bounds for each scattering length are simply six additional constraints to add to the many unitarity conditions we have already. We repeat the procedure above of maximizing and minimizing the value

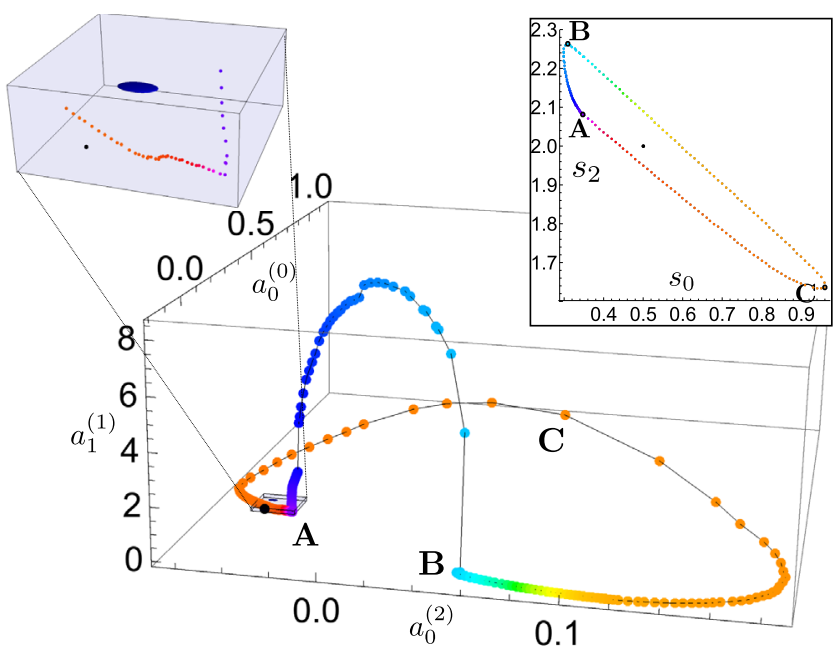

FIG. 4. Scattering lengths orbit around the lake. The colors in the inset and in the panel match in order to help following the orbit as we move around the lake. In the left inset we show a closeup off the $A$ kink: the black dot corresponds to the tree-level chiral theory and the ellipsoid to the experimental values for QCD. All the curves shown are obtained at fixed $N_{\max }=14$. The small nonsmoothness of the $A-B$ arc is a numerical artefact (in any case it occurs in a region where $a_{1}^{(1)}$ is huge, far from the pion physics we are interested in). 


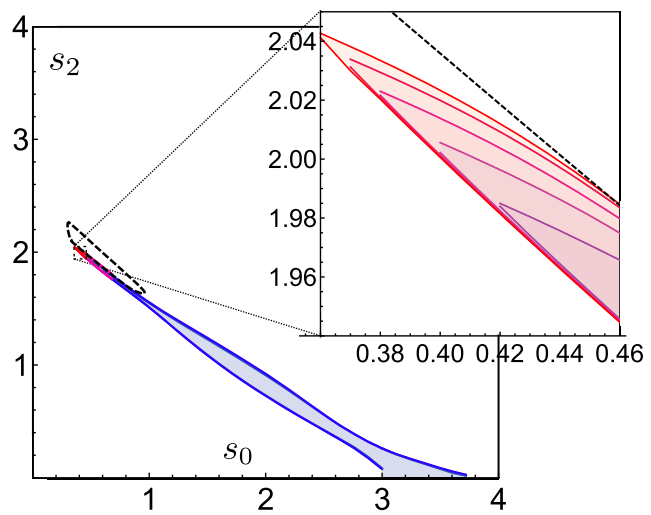

FIG. 5. Pion peninsula: we fix the $\rho$ resonance and the inequalities given by the experimental intervals for the three scattering lengths. The colored region is the one allowed by unitarity and different colors correspond to different $N_{\max }$ from 12 to 20 as we go from blue to red. The dashed contour encloses the pion lake.

of the amplitude in the symmetric channel $\mathcal{T}_{0}^{(2)}(s)$ with a zero $s_{0}$ imposed in the singlet channel $\mathcal{T}_{0}^{(0)}(s)$. The schematic representation of the result is in Fig. 2(b). We note that while before we excluded a small segment, now the maximum and minimum conditions exclude all but a tiny region for possible positions $s_{2}$ of the second chiral zero since only in a very small segment is the minimum negative and the maximum positive. Scanning over various $s_{0}$ we thus construct a full region in the $\left(s_{0}, s_{2}\right)$ plane which is now mostly excluded. It is represented in Fig. 5 and dubbed as the pion peninsula for obvious reasons.

It would be very interesting to see how the excluded region morphs into the lake in the limit when the inequalities imposed on the scattering lengths become looser.

In Fig. 6 we plot the effective ranges $b_{0}^{(0)}, b_{0}^{(2)}$ as we move around the peninsula boundary. Very nicely, we note that at the tip of the peninsula the orbit passes close to their experimental values in Table I. Besides, we observe the emergence of a first zero in $S_{0}^{(0)}$ that we identify as the $\sigma$ resonance. It too approaches its experimental position as we go to the tip of the peninsula.

The kink.-In this concluding section we fix the chiral zeros to the tip of the peninsula at our largest $N_{\max }=20$ value as illustrated in Fig. 5. We impose nothing else.

With these chiral zeros fixed, we repeated the analysis of the possible allowed values for the three scattering lengths, as in Fig. 1. We observed that with these extra constraints the resulting space is much more interesting and intricate.

There seem to be, for example, two cusps in this threedimensional space (one corresponding to the free theory and another with scattering lengths roughly twice as large as those measured in nature). The two cusps are connected by a sharp edge where the QCD scattering lengths seems to lie. To illustrate this, we consider in Fig. 7 a two-dimensional section of the allowed space intersecting this sharp edge at fixed $a_{0}^{(0)}=0.2196$, the expected

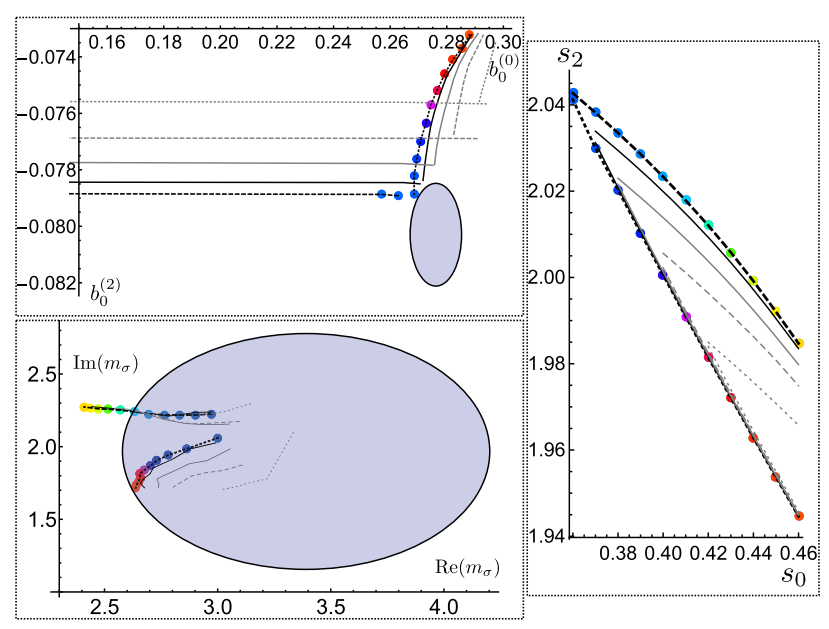

FIG. 6. Trajectory of the effective ranges in the $\left(b_{0}^{(0)}, b_{0}^{(2)}\right)$ plane (upper left panel) and of the spin zero in the $\left[\operatorname{Re}\left(m_{\sigma}\right), \operatorname{Im}\left(m_{\sigma}\right)\right]$ plane (lower left panel) as we travel the boundary of the peninsula (right) at fixed $N_{\max }=20$. Blue elliptic regions represent the experimental values and their uncertainty. In gray from lighter to darker the same trajectories are shown for $N_{\max }=16, \ldots, 19$.

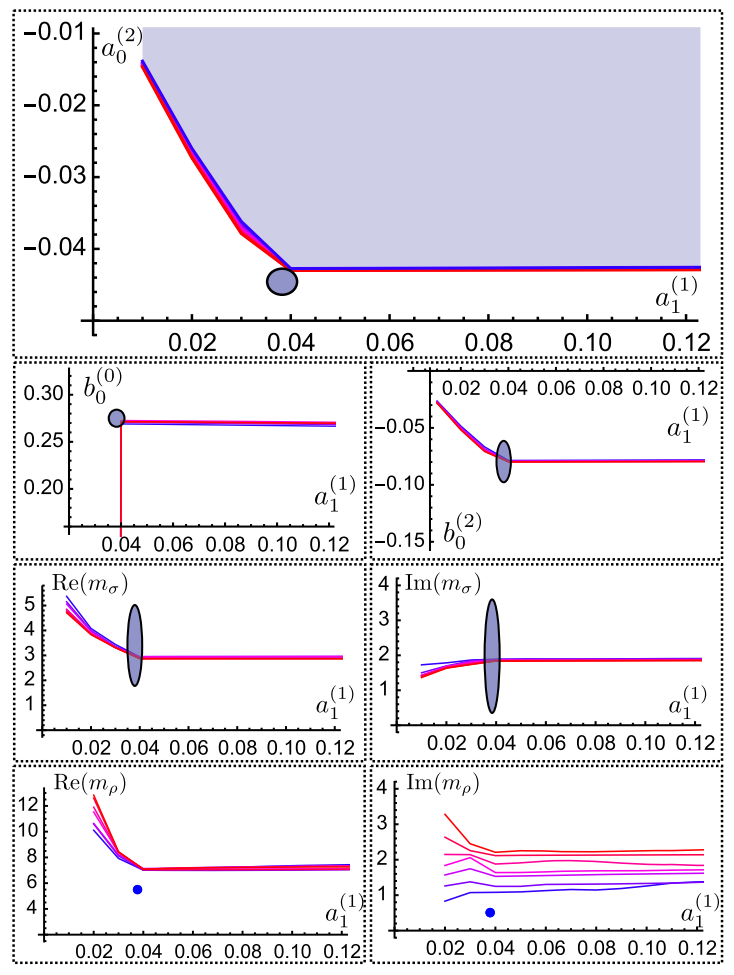

FIG. 7. Top-right panel: allowed region in the $\left(a_{0}^{(2)}, a_{1}^{(1)}\right)$ plane at fixed $s_{0}=0.36, s_{2}=2.04$ and $a_{0}^{(0)}=0.2196$. All the other panels show the effective ranges and the resonance masses along the same boundary as a function of $a_{1}^{(1)}$. Their experimental values lie in the blue ellipses. $N_{\max }$ goes from 14 to 20 (blue to red). 
experimental value for this scattering length. Not only do we have a very sharp kink but its location is extremely close to the experimental values.

Moreover, if we follow other quantities as the effective ranges $b_{0}^{(0)}, b_{0}^{(2)}$ or the complex mass $m_{\sigma}$ of the emerging spin-zero resonance as a function of $a_{1}^{(1)}$, they all show a kink compatible with their experimental values. We even observe the emergence of a zero in $S_{1}^{(1)}$ which we would like to identify with the $\rho$ resonance. (Its real part is actually quite close to the experimental one but its imaginary part is off [26].)

Conclusions.-One of the main outcomes of the discussion contained in this Letter is the constraint on the position of the chiral zeros in $\pi$ - $\pi$ scattering, obtained in a consistent way using our setup; see Fig. 5. An obvious next question which we are currently exploring is whether imposing the chiral zeros in the allowed region is enough to select a theory with phase shifts resembling the experimental ones and from which we can extract a resonance spectrum compatible with the physical one.

It would be also interesting to extend this study to the case of massless pions, i.e., exact Goldstone bosons and to other interesting setups with some symmetry breaking pattern, both in four or lower dimensions. [In two dimensions, the $O(N)$ bootstrap was recently addressed in [27-29] where contact with known integrable models was made.]

We would like to thank N. Arkani-Hamed, C. Bercini, M. Bianchi, L. Cordova, S. Frautschi, D. Gaiotto, A. Homrich, S. Komatsu, M. Kruczenski, M. Paulos, B. van Rees, and K. Zarembo for discussions. We wish to thank J. Toledo for collaboration in the early stage of this work. We are indebted to J. R. Pelaez for sharing with us the experimental phase shifts for $\pi-\pi$ scattering. We thank G. Colangelo, J.R. Pelaez, and A. Pilloni for useful comments on the draft. P. V. is specially grateful to Clay Riddell for enlightening conversations. Research at the Perimeter Institute is supported in part by the Government of Canada through NSERC and by the Province of Ontario through MRI. This research received funding from the Simons Foundation Grants No. 488649 (J. P.) and No. 488661 (P. V.) (Simons Collaboration on the Nonperturbative Bootstrap) and FAPESP Grants No. 2016/01343-7 and No. 2017/03303-1. J. P. is supported by the National Centre of Competence in Research SwissMAP funded by the Swiss National Science Foundation.

[1] M. F. Paulos, J. Penedones, J. Toledo, B. C. van Rees, and P. Vieira, arXiv:1708.06765.

[2] Other works used conformal mappings to parametrize fixed partial wave amplitudes [3,4].
[3] R. Garcia-Martin, R. Kaminski, J. R. Pelaez, J. Ruiz de Elvira, and F. J. Yndurain, Phys. Rev. D 83, 074004 (2011).

[4] I. Caprini, Phys. Rev. D 77, 114019 (2008).

[5] In practice, we bound all the spins up to $\ell_{\max }=20$ for the highest $N_{\max }$ we use. The asymptotic large-spin behaviour is also bounded. Our setup here is quite distinct from other treatments-based on Roy equations [6] for instance-which typically include the very first few spins only.

[6] B. Ananthanarayan, G. Colangelo, J. Gasser, and H. Leutwyler, Phys. Rep. 353, 207 (2001).

[7] M. Tanabashi et al. (Particle Data Group), Phys. Rev. D 98 , 030001 (2018).

[8] J. R. Pelaez, Phys. Rep. 658, 1 (2016).

[9] I. Caprini, G. Colangelo, and H. Leutwyler, Phys. Rev. Lett. 96, 132001 (2006).

[10] G. Colangelo, J. Gasser, and H. Leutwyler, Nucl. Phys. B603, 125 (2001).

[11] Unitarity from $4 m_{\pi}^{2}$ until the first inelastic threshold must be exactly saturated. In that range $S_{\ell}^{(I)}(s) \hat{S}_{\ell}^{(I)}(s)=1$, where $\hat{S}$ stands for $S$ in the second sheet. This functional relation can be analytically continued everywhere. As such (3) states that a resonance corresponds to a pole in the second sheet. A similar discussion for $1+1$ dimensions is in [12].

[12] N. Doroud and J. Elias Miro, J. High Energy Phys. 09 (2018) 052.

[13] S. L. Adler, Phys. Rev. 137, B1022 (1965).

[14] S. Weinberg, Phys. Rev. Lett. 17, 616 (1966).

[15] M. M. Nagels, T. A. Rijken, J. J. De Swart, G. C. Oades, J. L. Petersen, A. C. Irving, C. Jarlskog, W. Pfeil, H. Pilkuhn, and H. P. Jakob, Nucl. Phys. B147, 189 (1979).

[16] J. R. Batley et al. (NA48-2 Collaboration), Eur. Phys. J. C 70, 635 (2010).

[17] E. P. Wigner, Phys. Rev. 98, 145 (1955).

[18] Similar (but not identical) bounds were previously explored in [19-22].

[19] C. Lopez and G. Mennessier, Nucl. Phys. B96, 515 (1975).

[20] C. Lopez, Lett. Nuovo Cimento 13, 69 (1975).

[21] C. Lopez, Nucl. Phys. B88, 358 (1975).

[22] I. Caprini and P. Dita, J. Phys. A 13, 1265 (1980).

[23] The condition $\mathcal{T}_{0}^{(0)}\left(s_{0}\right)=0$ sets one linear constraint on the constants $a_{n m}$ and $b_{n m}$ in the ansatz (2) which allows us to solve for one of these constants. The resonance condition eliminates two more constants from the real and imaginary part of Eq. (3).

[24] S. El-Showk and M. F. Paulos, Phys. Rev. Lett. 111, 241601 (2013).

[25] It would be interesting to make the connection with the large $N$ limit, scaling accordingly the width of the $\rho$ resonance we impose.

[26] In our setup, the boundary of our $S$-matrix theory space will typically saturate unitarity. However, at some high energy we have sizeable inelasticities in pion scattering most notably at the $K \bar{K}$ threshold. As an instructive exploration we imposed-by hand-reasonable inelasticity in the unitarity constraints starting at this threshold and observed that most of the low energy parameters such as the ones plotted here are stable and almost do not change. (Similar games 
were played in two dimensions in [27] with similar conclusions.)

[27] L. Còrdova and P. Vieira, J. High Energy Phys. 12 (2018) 063.
[28] Y. He, A. Irrgang, and M. Kruczenski, J. High Energy Phys. 11 (2018) 093.

[29] M. F. Paulos and Z. Zheng, arXiv:1805.11429. 